\title{
Effect of BMAP-28 on human thyroid cancer TT cells is mediated by inducing apoptosis
}

\author{
DAQI ZHANG ${ }^{1 *}$, LANLAN WAN $^{2 *}$, JINNAN ZHANG $^{3}$, CHANG LIU $^{4}$ and HUI SUN ${ }^{1}$ \\ ${ }^{1}$ Division of Thyroid Surgery, Jilin Provincial Key Laboratory of Surgical Translational Medicine, \\ China-Japan Union Hospital of Jilin Univeristy; ${ }^{2}$ Department of Anesthesiology, The Second Hospital of Jilin University, \\ Jilin University School of Pharmaceutical Sciences; ${ }^{3}$ Department of Neurosurgery, \\ China-Japan Union Hospital of Jilin University, Changchun, Jilin 130033; ${ }^{4}$ School of Life Science and Technology, \\ China Pharmaceutical University, Nanjing, Jiangsu 210009, P.R. China
}

Received October 5, 2014; Accepted June 25, 2015

DOI: $10.3892 / \mathrm{ol} .2015 .3612$

\begin{abstract}
Thyroid cancer is the most common malignant endocrine tumor, with significant morbidity and mortality. Bovine myeloid antimicrobial peptide 28 (BMAP-28) is a cathelicidin that is found in bovine neutrophils. In the present study, the effect and relative mechanism of BMAP-28 on the human thyroid cancer TT cell line in vitro and in vivo were investigated. A 3-(4,5-dimethylthiazol-2-yl)-2,5-diphenyltetrazolium bromide assay, flow cytometry and a TT-xenograft mouse model were used in this study. The data obtained indicated that BMAP-28 significantly inhibited the proliferation of the TT cells in vitro. In addition, the Annexin V-fluorescein isothiocyanate/propidium iodide assay detected that BMAP-28 induced apoptotic effects in the TT cells. Moreover, the expression of activated caspase- 3 and -9 was upregulated at the transcriptional and translational levels. Simultaneously, the expression of matrix metalloproteinase (MMP)3 and MMP9 was downregulated following BMAP-28 treatment. Finally, BMAP-28 significantly prevented the tumor growth in the TT-xenograft mouse model. These results indicated that BMAP-28 could be a potential agent for the treatment of thyroid cancer.
\end{abstract}

\section{Introduction}

Thyroid cancer is the most common malignant endocrine tumor, with significant morbidity and mortality $(1,2)$. A steady increase

Correspondence to: Professor Hui Sun, Division of Thyroid Surgery, Jilin Provincial Key Laboratory of Surgical Translational Medicine, China-Japan Union Hospital of Jilin University, 126 Xiantai Boulevard, Changchun, Jilin 130033, P.R. China

E-mail: sun_huijilin@163.com

*Contributed equally

Key words: BMAP-28, thyroid cancer, TT cells, apoptosis, caspase-3, caspase-9, matrix metalloproteinase 3, matrix metalloproteinase 9 in the incidence of this cancer type has been recorded over the last three decades (3). It is known that the female North American population has the highest incidence of thyroid cancer, with an age-standardized rate of 15.1 per 100,000 females. In Western Europe, the age-standardized rate is 5.8 per 100,000 females (4). Thyroid cancer comprises tumors with different clinical and histological features (5). Specifically, papillary and follicular thyroid cancers are slow-growing and well-differentiated. However, anaplastic thyroid cancers are undifferentiated neoplasias with much more aggressive activity, usually leading to patient mortality within one year of diagnosis (6).

Antimicrobial peptides (AMPs) are naturally occurring molecules found in microorganisms, plants and animals, and are considered as an essential component in the innate immunity $(7,8)$. Despite their structural conservation, AMPs are emerging as a novel source of molecules and have a broad spectrum of activity, such as antibacterial, antifungal and antiviral functions (9). More recently, their abilities against tumor cells have been confirmed by selectively killing cancer cells, whose membrane proteins are negatively charged through glycosylation (10). AMPs exert anticancer activity by a membranolytic mode of action or via interaction with intracellular targets, or through use of a combination of the two (11). Cationic antitumor peptides represent potential antitumor therapy agents, as they have a number of advantages compared with other chemical agents, including a comparatively simple structure, a low molecular mass, few adverse reactions, greater specific cytotoxicity to tumor cells over healthy cells, ease of absorption and a low risk for the induction of multi-drug resistance (12-14).

Bovine myeloid antimicrobial peptide 28 (BMAP-28) is a cathelicidin, which is found in bovine neutrophils (15). It has been reported that BMAP-28 had a wide range of antimicrobial activities and could confer protection to bacterial infection and sepsis in animals $(16,17)$. Additionally, previous studies found that BMAP-28 exhibited significantly reduced cytotoxicity against 3T3 cells and erythrocytes (18). However, the antitumor effect and clear mechanism of BMAP-28 on thyroid cancer cells have not yet been investigated.

In the present study, the activity and mechanism of BMAP-28 on human thyroid cancer TT cells were investigated in order to highlight the therapeutic potential of BMAP-28 in thyroid cancer. 


\section{Materials and methods}

Regents. BMAP-28 (GGLRSLGRKILRAWKKYGPIIVPIIRIG) was synthesized using solid-phase methodology at GL Biochemistry Corporation (Shanghai, China). The resulting peptide was purified by preparative reverse-phase high-performance liquid chromatography, resulting in final products that were deemed $>95 \%$ pure. Synthetic antimicrobial peptide was reconstituted in phosphate-buffered saline (PBS; pH 7.4) for subsequent experiments. 3-(4,5-Dimethylthiazol-2-yl)-2,5-diphenyltetrazolium bromide (MTT), sodium pyruvate and dimethyl sulfoxide (DMSO) were obtained from Sigma-Aldrich (St. Louis, MO, USA). Annexin V-fluorescein isothiocyanate/propidium iodide (Annexin V-FITC/PI) were purchased from Mbchemic (Shanghai, China). Colorimetric assay kits for detection of capase-3 and -9 were obtained from BioVision (Mountain View, CA, USA). Anti-human matrix metalloproteinase (MMP)-3 phycoerythrin mouse monoclonal antibody (MAb; 1:1,000) and anti-human MMP-9 fluorescein rabbit MAb $(1: 1,000)$ were purchased from R\&D Systems Inc. (Minneapolis, MN, USA). The Easy Plus Mini kit, iScript Select cDNA Synthesis kit, SyberGreen qPCR Primer and iCycler iQ Multicolor Real-Time PCR Detection System were all purchased from KeyGEN (Nanjing, China). The study was approved by the ethics committee of the Department of Thyroid Surgery, China-Japan Union Hospital of Jilin University (Changchun, China).

Cell culture. Human thyroid cancer TT cells were obtained from the American Type Culture Collection (Manassas, VA, USA) and maintained as previously described (19). Briefly, the TT cells were maintained in F-12K supplemented with $16 \%$ fetal bovine serum (Gibco Life Technologies, Carlsbad, CA, USA), $100 \mathrm{U} / \mathrm{ml}$ penicillin and $100 \mathrm{U} / \mathrm{ml}$ streptomycin. The cells were cultured at $37^{\circ} \mathrm{C}$ in a humidified incubator containing $5 \% \mathrm{CO}_{2}$.

MTT assay. An MTT assay was performed to evaluate the effect of BMAP-28 on the TT cells. As previously described (20), the cells were seeded in 96-well plates at a concentration of $5 \times 10^{3}$ cells/well with F-12K medium containing $10 \%$ fetal calf serum and allowed to attach for $12 \mathrm{~h}$. Different concentrations of BMAP-28 $(0,0.5,1,2,4,8,16$ and $32 \mu \mathrm{M})$ were added to the cells and further incubated for 24,48 or 72 h. Following incubation, the cells were incubated with MTT solution $(5 \mu \mathrm{g} / \mathrm{ml})$ for $4 \mathrm{~h}$ at $37^{\circ} \mathrm{C}$, then switched into $150 \mu \mathrm{l} \mathrm{DMSO}$ and shaken for $5 \mathrm{~min}$ at room temperature prior to measuring absorbance at $490 \mathrm{~nm}$.

Cell apoptosis assay. Cell apoptosis assays (21) were conducted by double staining using Annexin V and PI kits (eBioscience Inc., San Diego, CA, USA) to investigate whether BMAP-28 induces apoptosis in TT cells. The TT cells were seeded in 6 -well plastic plates $\left(5 \times 10^{5}\right.$ cells/well) $24 \mathrm{~h}$ prior to BMAP-28 treatment. The medium supernatant in the plates was then replaced, and various concentrations of BMAP-28 (ranging from 0 to $4 \mu \mathrm{M}$ ), diluted in $\mathrm{PBS}$, were added to the plates. Subsequent to incubation at $37^{\circ} \mathrm{C}$ in a humidified atmosphere with $5 \% \mathrm{CO}_{2}$ for $48 \mathrm{~h}$, the cells were harvested and washed twice with PBS. For the Annexin V-FITC/PI assay, the cells were treated according to the manufacturer's instructions. Briefly, cells were suspended in $100 \mu \mathrm{l}$ binding buffer (eBioscience, Inc.) and $5 \mu 1$ Annexin V-FITC. This mixture was incubated at room temperature in the dark for

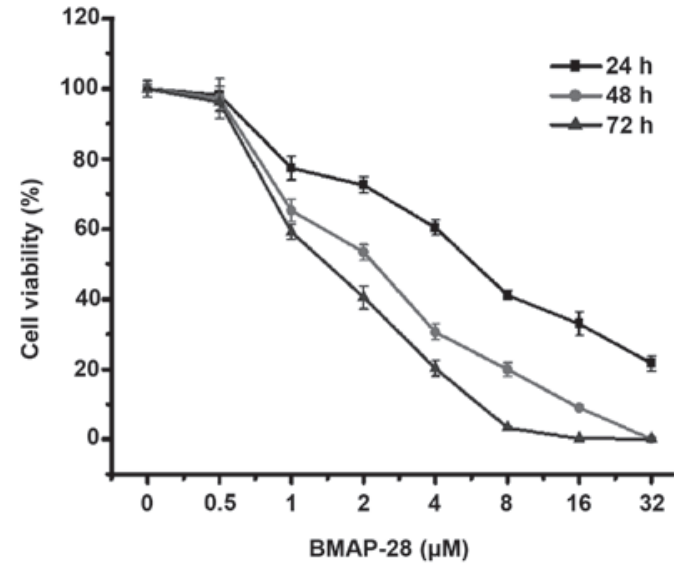

Figure 1. Bovine myeloid antimicrobial peptide 28 (BMAP-28) inhibits the proliferation of human thyroid cancer TT cells. The TT cells were treated with different concentrations of BMAP-28 (ranging from 0 to $32 \mu \mathrm{M}$ ) for the indicated time periods $(24,48$ and $72 \mathrm{~h}$ ), and then the cell viability was measured by 3-(4,5-dimethylthiazol-2-yl)-2,5-diphenyltetrazolium bromide assay. The BMAP-28 treatment group exhibited inhibited cell proliferation in a dose- and time-dependent manner. Data are shown as the mean \pm standard deviation from three separate determinations with four replicates each.

15 min. Next, cells were washed once with binding buffer, suspended in $100 \mu \mathrm{l}$ binding buffer and $5 \mu \mathrm{l}$ PI was added. Following this, the cells were analyzed using a BD FACS Calibur flow cytometer (BD Biosciences, San Jose, CA, USA). Signals of Annexin V-FITC were measured at $488 \mathrm{~nm}$ for excitation and $525 \mathrm{~nm}$ for emission, and signals of PI were detected at $535 \mathrm{~nm}$ for excitation and $615 \mathrm{~nm}$ for emission.

Detection of caspase-3, caspase-9, MMP3 and MMP9 expression. Caspase-3, caspase-9, MMP3 and MMP9 expression was assessed using colorimetric assay kits according to the manufacturer's instructions (22). The TT cells were treated with various concentrations of BMAP-28 (ranging from 0 to $4 \mu \mathrm{M}$ ) for $24 \mathrm{~h}$. Next, cell pellets were washed twice with PBS and scrapped with ice-cold lysis buffer. Active caspase 3, caspase 9, MMP3 and MMP9 expression was measured accordingly: The fluorescence intensity was measured at $405 \mathrm{~nm}$ with a flow cytometer to detect the production of active molecules.

Quantitative polymerase chain reaction ( $q P C R)$. The effect of BMAP-28 on the caspase 3, caspase 9, MMP3 and MMP9 RNA expression of the TT cells was examined by a qPCR, as previously described (23). Total RNA was extracted from the TT cells with or without BMAP-28 (ranging from 0 to $4 \mu \mathrm{M}$ ) treatment using TRIzol reagent and then purified with an RNeasy Mini kit (Qiagen Sciences Inc., Germantown, MD, USA). Next, qPCR was performed in an ABI PRISM 7300 sequence detection system (Applied Biosystems Life Technologies, Foster City, CA, USA), with 3 wells (10 $\mu$ l/well) set for every reaction. The reactions were performed under the following conditions: $95^{\circ} \mathrm{C}$ for $10 \mathrm{~min} ; 45$ cycles of $95^{\circ} \mathrm{C}$ for $15 \mathrm{sec}, 60^{\circ} \mathrm{C}$ for $30 \mathrm{sec}$ and $72^{\circ} \mathrm{C}$ for $30 \mathrm{sec}$ to collect signals; followed by $72^{\circ} \mathrm{C}$ for 7 min to obtain a solubility curve. Relative mRNA expression was calculated using the comparative CT $\left(2^{-\Delta \Delta C t}\right)$ method.

The sequences of the primers were as follows: Caspase 3 forward, 5'-AGGAAGGTGGCAACG-3' and reverse, 5'-CGC CAAATCTTGCTAAT-3'; caspase 9 forward, 
A

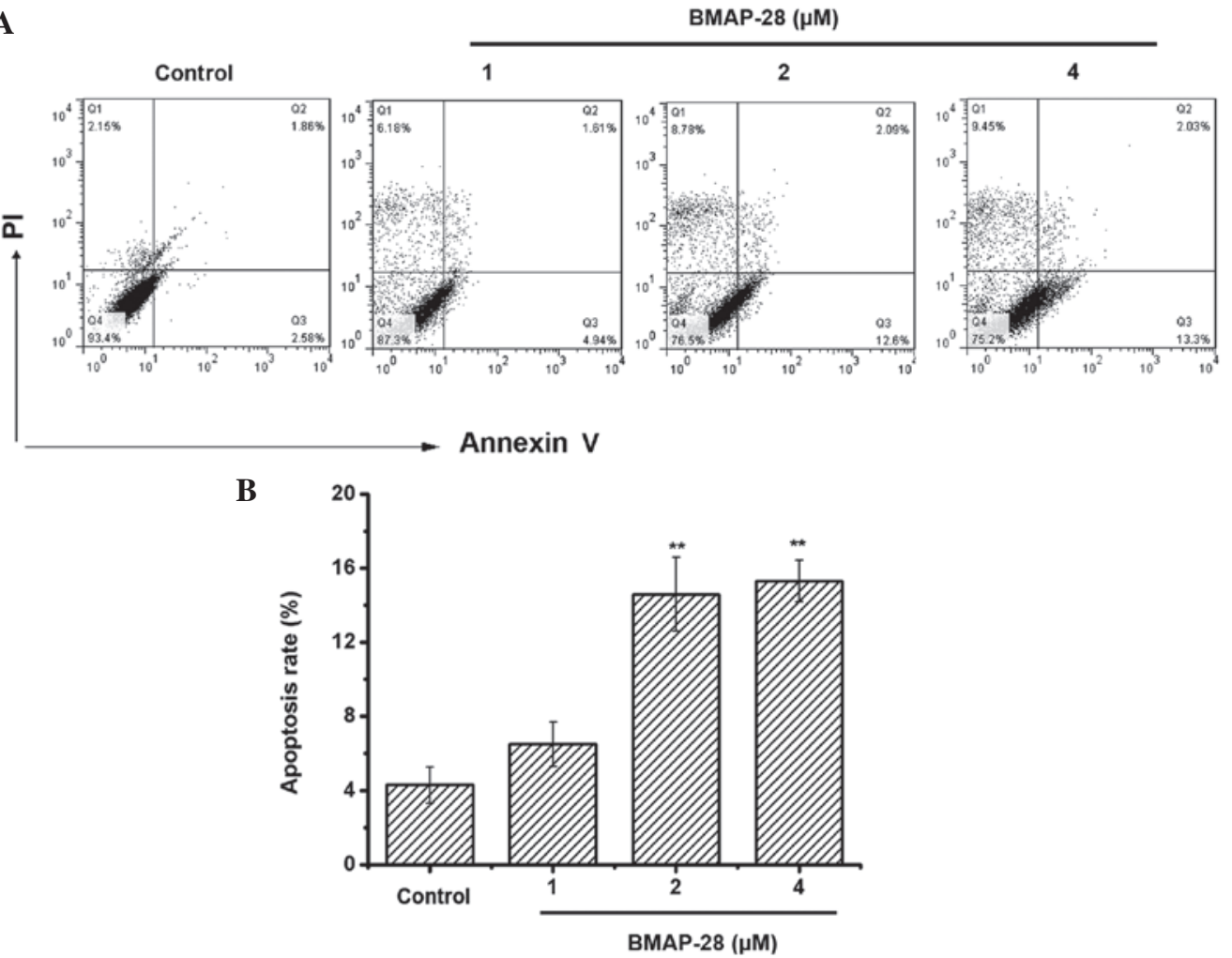

Figure 2. BMAP-28 induces the cell apoptosis of TT cells. (A) Analysis of TT cell apoptosis was conducted by flow cytometry using Annexin V-fluorescein isothiocyanate/PI staining. Late apoptotic cells, which could bind Annexin V and PI, are in the upper right quadrant, while early apoptotic cells, which could bind Annexin V, are in the lower right quadrant. Representative dot plots indicate the distribution of different cell populations following treatment with BMAP-28 (ranging from 0 to $4 \mu \mathrm{M}$ ) for $48 \mathrm{~h}$. (B) Percentage of apoptosis. Respective data from at least three independent experiments are summarized and presented as the mean \pm standard deviation ( $(*$ P $<0.01$ vs. control group). BMAP-28, bovine myeloid antimicrobial peptide 28; PI, propidium iodide.

5'-GGCTGTCTA CGGCACAGATGGA-3' and reverse, 5'-CTGGCTCGGGGT TACTGCCAG-3'; MMP-3 forward, 5'-CTCACAGACCTGACT CGGTT-3' and reverse, 5'-CACGCCTGAAGGAAGAGATG-3'; and MMP-9 forward, 5'-CGCAGACATCGTCATGT-3' and reverse, 5'-GGATTGGCCTTGGAAGATGA-3'.

TT-xenograft mouse model and BMAP-28 administration in vivo. Nude mice purchased from the Academy of Military Medical Science (Beijing, China) were housed in a rodent facility at $22 \pm 1^{\circ} \mathrm{C}$ with a 12 -h light-dark cycle and provided with continuous standard rodent chow and water for acclimatization. The TT cells $\left(2 \times 10^{7}\right.$ cells $\left./ \mathrm{ml} ; 0.1 \mathrm{ml}\right)$ were inoculated intradermally into the hind flank. When the diameter of the tumors reached $0.5 \mathrm{~cm}$, the mice were randomly divided into four groups ( $\mathrm{n}=6$ per group) consisting of a model control group administered with normal saline, and three BMAP-28-treated groups administered at $0.25,0.50$ or $1 \mathrm{mg} / \mathrm{kg}$ body weight These four groups were administered intra-tumoral injections $(50 \mu \mathrm{l})$ once every 5 days for 25 days. At the indicated time-points, the mice were sacrificed by cervical dislocation $24 \mathrm{~h}$ after the final administration. The tumor weights of the mice from each group were measured. During the treatment, the tumor volume (TV) of each mouse was measured.

The antitumor activity was expressed as the inhibitory rate (\%) and calculated as follows: (A - B) / A x 100, where $\mathrm{A}$ and $\mathrm{B}$ were the average tumor weight of the model and BMAP-28 groups, respectively.
The TV was calculated using the following formula: $\mathrm{TV}=1 / 2 \times \mathrm{a} \mathrm{x} \mathrm{b}^{2}$, where $\mathrm{a}$ and $\mathrm{b}$ are the long and short diameters of the tumors in each mouse, respectively.

Statistical analysis. All experiments were performed in triplicate. The data are presented as the mean \pm standard deviation. Statistical analysis was performed by a one-way analysis of variance, followed by Dunnett's multiple comparison or Student's t-test, where $\mathrm{P}<0.05$ was considered to indicate a statistically significant difference.

\section{Results}

BMAP-28 suppresses cell proliferation of TT cells. To clarify the effects of BMAP-28 on thyroid cancer cells in vitro, human thyroid cancer TT cells were treated with BMAP-28 at different concentrations (ranging from 0 to $32 \mu \mathrm{M}$ ) for 24,48 and $72 \mathrm{~h}$. As shown in Fig. 1, BMAP-28 time- and dose-dependently inhibited the cell proliferation. Specifically, at $48 \mathrm{~h}$, the cell viabilities were $65.2,53.3,30.7$, and $19.9 \%$ at the dose of 1,2 , 4 and $8 \mu \mathrm{M}$ BMAP-28, respectively. The results illustrate that BMAP-28 exerted a direct cytotoxic effect on the TT cells.

BMAP-28 induces cell apoptosis of TT cells. To test whether BMAP-28 induced cell death through apoptosis, an Annexin V-FITC/PI assay was performed to measure the percentage of apoptotic cells. Positive staining with Annexin V-FITC correlated with the loss of membrane 
A

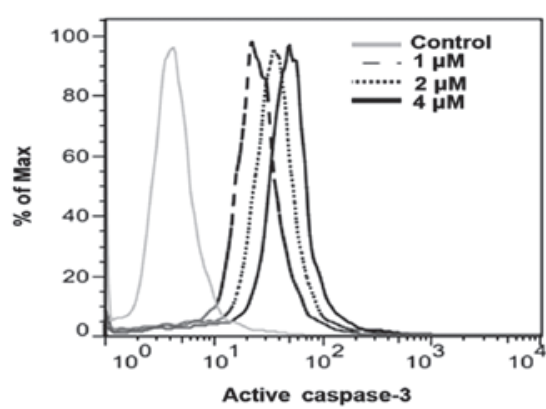

C

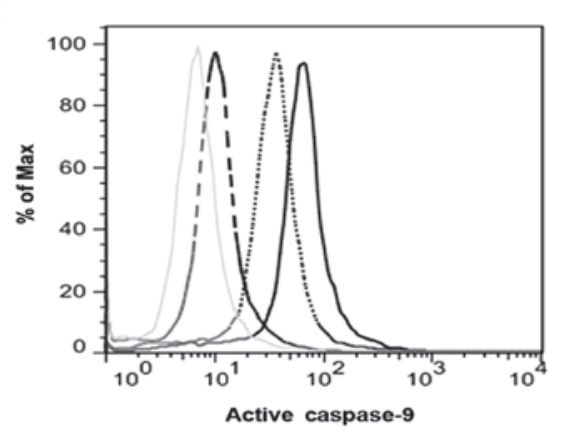

E

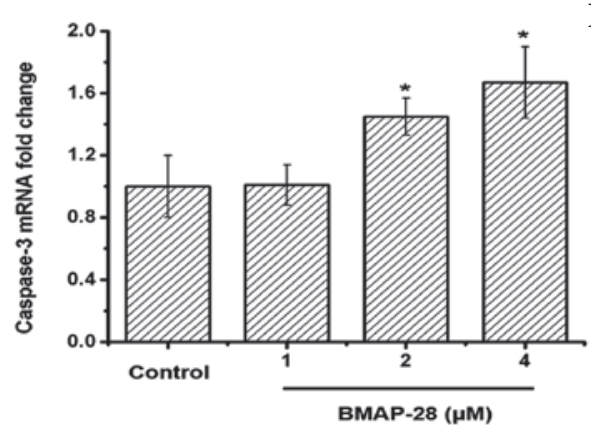

B

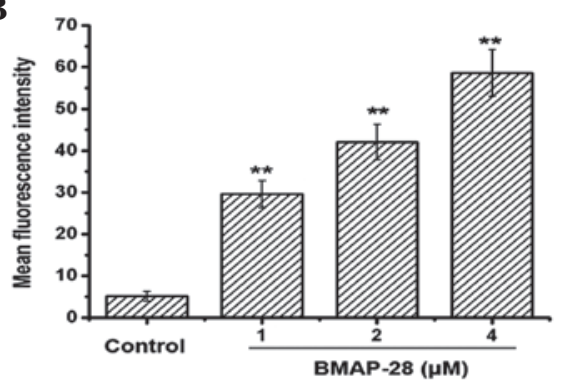

D
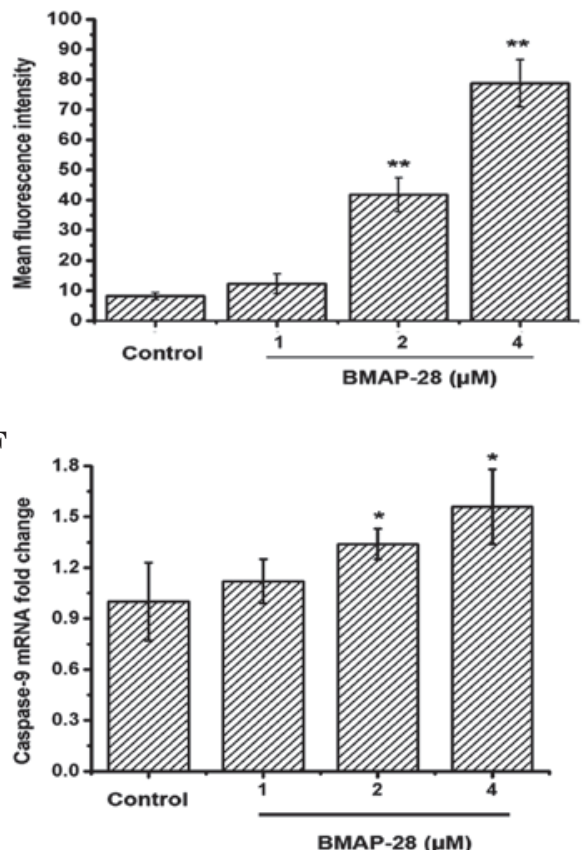

Figure 3. Bovine myeloid antimicrobial peptide 28 (BMAP-28) activates caspase-3 and caspase-9 in TT cells. The TT cells were cultured in the presence of different concentrations of BMAP-28 (ranging from 0 to $4 \mu \mathrm{M}$ ) for $48 \mathrm{~h}$ and subjected to the quantitative polymerase chain reaction and flow cytometric analysis for active caspase-3 and caspase-9, respectively. BMAP-28 exhibited an initial increase in the level of (A and B) active caspase-3 and (C and D) active caspase-9. The histogram overlays shown in (A) and (C) are representative of triplicates, and the combined results are presented in (B) and (D). BMAP-28 upregulated (E) caspase-3 and $(\mathrm{F})$ caspase-9 gene expression $\left(\mathrm{n}=3\right.$; data are shown as the mean \pm standard deviation. ${ }^{*} \mathrm{P}<0.05$, ${ }^{* * *} \mathrm{P}<0.01$ vs. control group).

polarity, among which the complete loss of membrane integrity lead to apoptosis. PI entered the cells after the loss of membrane integrity. Therefore, dual labeling with Annexin V and PI was adopted for discriminating between unaffected and apoptotic cells. The results indicated that BMAP-28 induced apoptotic effects on the TT cells (Fig. 2A). Specifically, the induced apoptotic cell accumulation reached 6.5, 14.6 and $15.3 \%$ at the dose of 1,2 and $4 \mu \mathrm{M}$ BMAP-28, respectively (Fig. 2B). Therefore, induced apoptosis could be one mechanism for BMAP-28 in preventing the proliferation of TT cells.

BMAP-28 activates caspase-3 and caspase-9 in TT cells. Given that caspase- 3 and caspase- 9 are activated during the early stage of apoptosis, they are treated as markers of apoptotic cells (22). The present results indicated that caspase-3 and caspase-9 were activated in the BMAP-28-treated TT cells as the dose increased (Fig. 3A-D). Moreover, similar results were obtained in the qPCR assay where the RNA levels of caspase-3 and caspase- 9 in the TT cells were increased after exposure to BMAP-28 (Fig. 3E and F).
BMAP-28 suppresses MMP3 and MMP9 in TT cells. To investigate whether BMAP-28 could affect MMP3 and/or MMP9, further experiments were performed. The obtained data indicated that the protein and mRNA expression of MMP3 and MMP9 in the TT cells was downregulated in a dose-dependent manner following exposure to BMAP-28 (Fig. 4).

BMAP-28 prevents tumor growth in the TT-xenograft mouse model. In order to detect whether BMAP-28 could prevent the TT growth in vivo, the TT-xenograft nude mouse model was established. As shown in Fig. 5, BMAP-28 significantly inhibited the TT cell growth as the dose increased. Specifically, in the $1 \mathrm{mg} / \mathrm{kg}$ group, the tumor inhibitory rate was up to $55.6 \%$. These results suggested that BMAP-28 administration prevented the TT tumor growth in the mice.

\section{Discussion}

Thyroid cancers are the most commonly occurring neoplasms of the endocrine system, with an excellent prognosis in the initial stages (1). The cancer is histopathologically 


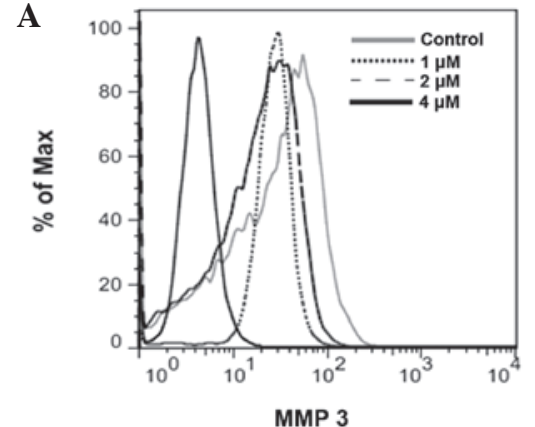

C

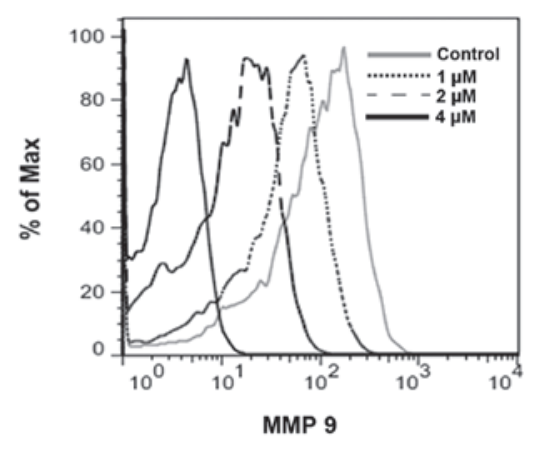

$\mathbf{E}$

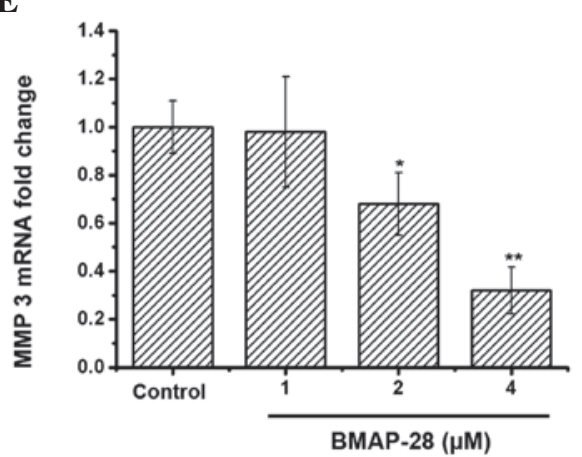

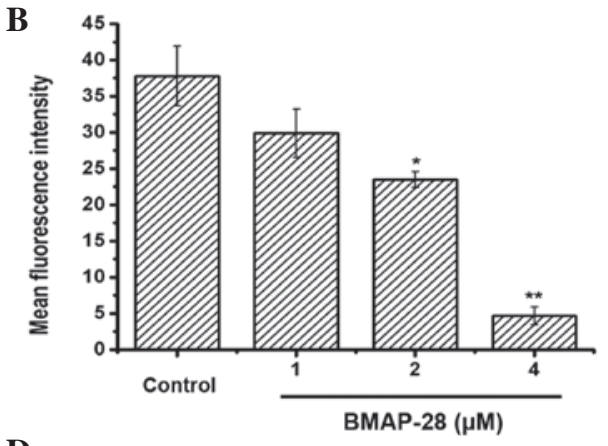

D

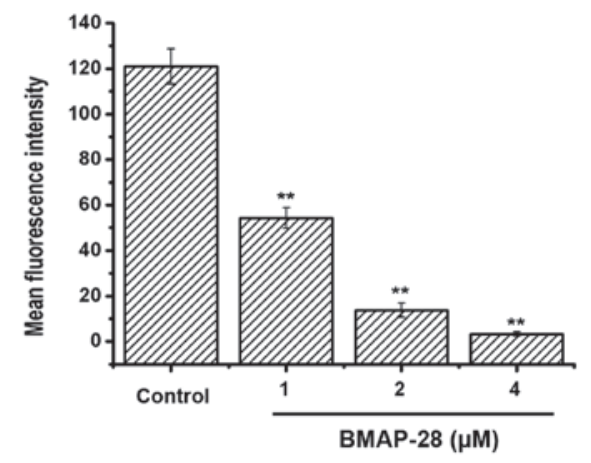

$\mathbf{F}$

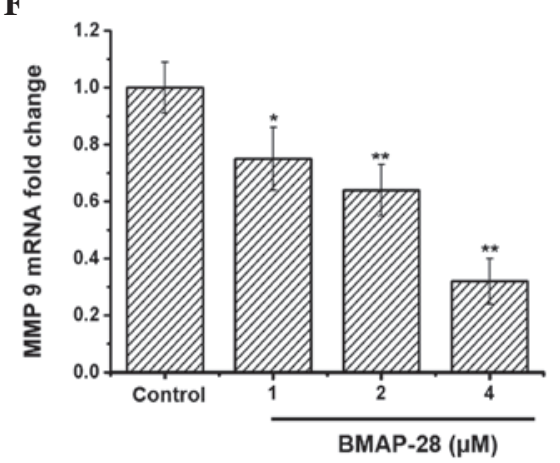

Figure 4. Bovine myeloid antimicrobial peptide 28 (BMAP-28) prevents the transcription and translation of matrix metalloproteinase (MMP) 3 and MMP9. (A-D) BMAP-28 suppressed the protein expression of (A and B) MMP3 and (C and D) MMP9 in the TT cells. The TT cells were treated with BMAP-28 (ranging from 1 to $4 \mu \mathrm{M}$ ) for $48 \mathrm{~h}$, then the cells were collected and analyzed by flow cytometry. (E and F) Downregulation of MMP3 and MMP9 transcription in BMAP-28-treated TT cells. RNA extracted from TT cells treated with or without BMAP-28 $(1,2$ and $4 \mu \mathrm{M})$ for $48 \mathrm{~h}$ were analyzed by quantitative polymerase chain reaction, as described in the Materials and methods section. Values are presented as the mean \pm standard deviation from three separate experiments. ${ }^{*} \mathrm{P}<0.05$ and ${ }^{* *} \mathrm{P}<0.01$ vs. control group.
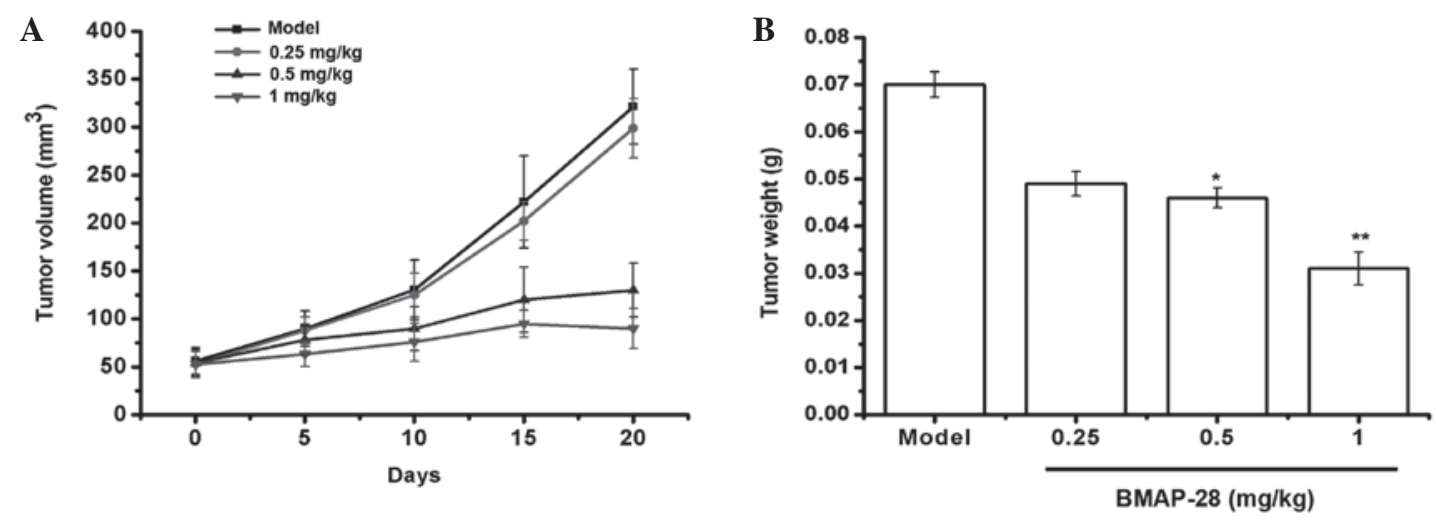

Figure 5. Bovine myeloid antimicrobial peptide 28 (BMAP-28) significantly suppresses tumor growth in vivo. (A) The tumor volume curve in the TT-bearing mice treated with or without BMAP-28. (B) The tumor weights were decreased in a dose-dependent manner. Data are presented as the mean \pm standard deviation. ${ }^{*} \mathrm{P}<0.05$ and ${ }^{* *} \mathrm{P}<0.01$ vs. the control group.

classified into three groups: Differentiated thyroid carcinoma (94\%), medullary thyroid carcinoma (4\%) and anaplastic (undifferentiated) thyroid carcinoma (2\%). However, there are few available therapeutic options for advanced or metastatic 
disease. Hence, it is necessary to develop novel drugs with high efficiency and low toxicity.

AMPs, which are effective molecules of innate immunity, have recently been shown to exhibit anticancer activity; they have gained much attention as potential anticancer agents (24). AMPs with cancer-selective toxicity have become a potential source of alternative chemotherapeutic agents that may overcome the limitations of current drugs.

BMAP-28, a cathelicidin found in bovine neutrophils, has been shown to possess broad-spectrum antimicrobial activity (16). However, its anti-thyroid cancer activity has not been elucidated. In the present study, the anticancer activity and mechanisms of BMAP-28 on human TT cells were investigated. It was found that BMAP-28 significantly inhibited the proliferation of the TT cells in vitro (Fig. 1), indicating that BMAP-28 possessed anti-TT cell activity. The mechanisms of BMAP-28 on the TT cells were investigated in the further experiments.

Apoptosis, an active process leading to cell death, is mediated by programmed signaling pathways that can be initiated by a range of types of extracellular or intracellular stimulation. In order to evaluate the efficacy of anticancer therapies, the ability to induce apoptosis is essential (25). In the present study, the Annexin V-FITC/PI double-staining assay suggested that BMAP-28 induced apoptosis in the TT cells (Fig. 2). Members of the caspase family have major roles in cell apoptosis (26). The caspase cleavage cascade is a well-known process that starts with initiator caspase activation via the intrinsic or extrinsic pathways. Caspase-9 is the key initiator caspase for the intrinsic pathway to induce cell death, and activation of this caspase occurs with the release of cytochrome $c$ at the mitochondrial membrane. Caspase 3 is an effector caspase that mediates the cleavage of multiple cellular proteins in tandem with other effector caspases (27). The present study found that the protein and mRNA expression of caspase- 3 and caspase- 9 increased significantly in the BMAP-28-treated group, which indicated that BMAP-28 could induce apoptosis in the TT cells partly via the activation of caspase- 9 and caspase-3 (Fig. 3). From these results, it may be concluded that activated caspase- 3 and -9 contribute to BMAP-28-induced apoptosis.

MMPs family members are among the best characterized proteases and consist of zinc endopeptidases sharing homologous catalytic domains. The main characteristic of these enzymes is to catalyze the cleavage of the extracellular matrix protein components and to function in tissue remodeling during development, wound healing, cancer invasion and metastasis, as well as other physiological processes (28). A wide variety of extracellular matrix components are known to be degraded by the MMP-9 and MMP-3 enzymes, including collagen I, collagen IV and the proteoglycans (29). MMP-9 has significant involvement in tumor metastasis due to its role in basement membrane cleavage, which allows migratory phenotype cells to be more motile and invasive (30). The inhibition of MMP-9 and MMP-3 expression may therefore be significant in the development of tumor metastasis treatments. As shown in Fig. 4, in the present study, BMAP-28 was able to inhibit the mRNA and protein of MMP-9 and MMP-3.

BAMP-28 exhibited a marked inhibitory effect on the TT cells in vitro, which contributed to the induction of apoptosis (caspase-3/9) and the inhibition of cancer invasion (MMP-3/9). TT-xenograft tumor models were established in nude mice (Fig. 5) to investigate whether BAMP-28 also had an inhibitory effect in vivo. Compared with the model control, BMAP-28 dose-dependently suppressed the proliferation of the TT cells in the tumor-bearing mice. Hence, BAMP-28 could inhibit the TT cell proliferation, not only in vitro, but also in vivo.

In summary, BMAP-28 inhibited the proliferation of human TT cells in vitro and in vivo through the induction of the activation of caspase- 3 and caspase- 9 and the inhibition of MMP-3 and MMP-9. These results suggested that BMAP-28 may be a potential candidate for the treatment of thyroid cancer.

\section{Acknowledgements}

The present study was financially supported by a grant from Jilin Provincial Finance Department (no. SCZSY201504).

\section{References}

1. Kleiman DA, Buitrago D, Crowley MJ, Beninato T, Veach AJ, Zanzonico PB, Jin M, Fahey TJ III and Zarnegar R: Thyroid stimulating hormone increases iodine uptake by thyroid cancer cells during BRAF silencing. J Surg Res 182: 85-93, 2013.

2. Hombach-Klonisch $\mathrm{S}$, Natarajan $\mathrm{S}$, Thanasupawat $\mathrm{T}$, Medapati M, Pathak A, Ghavami S and Klonisch T: Mechanisms of therapeutic resistance in cancer (stem) cells with emphasis on thyroid cancer cells. Front Endocrinol (Lausanne) 25: 5-37, 2014.

3. Pellegriti G, Frasca F, Regalbuto C, Squatrito S and Vigneri R: Worldwide increasing incidence of thyroid cancer: Update on epidemiology and risk factors. J Cancer Epidemiol 2013: 965212 , 2013.

4. Stjepanovic $\mathrm{N}$ and Capdevila $\mathrm{J}$ : Multikinase inhibitors in the treatment of thyroid cancer: Specific role of lenvatinib. Biologics 8: 129-139, 2014.

5. Massimino M, Vigneri P, Fallica M, Fidilio A, Aloisi A, Frasca F and Manzella L: IRF5 promotes the proliferation of human thyroid cancer cells. Mol Cancer 16: 11-21, 2012.

6. Fagin JA: Perspective: Lessons learned from molecular genetic studies of thyroid cancer-insights into pathogenesis and tumor-specific therapeutic targets. Endocrinology 143: 2025-2028, 2002.

7. Hancock RE and Chapple DS: Peptide antibiotics. Antimicrob Agents Chemother 43: 1317-1323, 1999.

8. Hancock RE and Sahl HG: Antimicrobial and host-defense peptides as new anti-infective therapeutic strategies. Nat Biotechnol 24: 1551-1557, 2006.

9. Mulder KC, Lima LA, Miranda VJ, Dias SC and Franco OL: Current scenario of peptide-based drugs: The key roles of cationic antitumor and antiviral peptides. Front Microbiol 4: 321, 2013.

10. Mader JS and Hoskin DW: Cationic antimicrobial peptides as novel cytotoxic agents for cancer treatment. Expert Opin Investig Drugs 15: 933-946, 2006.

11. Camilio KA, Berge G, Ravuri CS, Rekdal O and Sveinbjørnsson B: Complete regression and systemic protective immune responses obtained in B16 melanomas after treatment with LTX-315. Cancer Immunol Immunother 63: 601-613, 2014.

12. Schweizer F: Cationic amphiphilic peptides with cancer-selective toxicity. Eur J Pharmacol 625: 190-194, 2009.

13. Riedl S, Zweytick D and Lohner K: Membrane-active host defense peptides-challenges and perspectives for the development of novel anticancer drugs. Chem Phys Lipids 164: 766-781, 2011.

14. Liu S, Yang H, Wan L, Cheng J and Lu X: Penetratin-mediated delivery enhances the antitumor activity of the cationic antimicrobial peptide magainin II. Cancer Biother Radiopharm 28: 289-297, 2013.

15. Lynn MA, Kindrachuk J, Marr AK, Jenssen H, Panté N, Elliott MR, Napper S, Hancock RE and McMaster WR: Effect of BMAP-28 antimicrobial peptides on Leishmania major promastigote and amastigote growth: Role of leishmanolysin in Parasite Survival. PLoS Negl Trop Dis 5: e1141, 2011. 
16. Benincasa M, Skerlavaj B, Gennaro R, Pellegrini A and Zanetti M: In vitro and in vivo antimicrobial activity of two alpha-helical cathelicidin peptides and of their synthetic analogs. Peptides 24: 1723-1731, 2003.

17. Giacometti A, Cirioni O, Ghiselli R, Bergnach C, Orlando F, D'Amato G, Mocchegiani F, Silvestri C, Del Prete MS, Skerlavaj B, et al: The antimicrobial peptide BMAP-28 reduces lethality in mouse models of staphylococcal sepsis. Crit Care Med 32: 2485-2490, 2004.

18. Ahmad A, Asthana N, Azmi S, Srivastava RM, Pandey BK Yadav V and Ghosh JK: Structure-function study of cathelicidin-derived bovine antimicrobial peptide BMAP-28: Design of its cell-selective analogs by amino acid substitutions in the heptad repeat sequences. Biochim Biophys Acta 1788: 2411-2420, 2009.

19. Starenki D and Park JI: Mitochondria-targeted nitroxide Mito-CP, suppresses medullary thyroid carcinoma cell survival in vitro and in vivo. J Clin Endocrinol Metab 98: 1529-1540, 2013.

20. Wang H, Ke M, Tian Y, Wang J, Li B, Wang Y, Dou J and Zhou C: BF-30 selectively inhibits melanoma cell proliferation via cytoplasmic membrane permeabilization and DNA-binding in vitro and in B16F10-bearing mice. Eur J Pharmacol 707: $1-10,2013$

21. Tian Y, Wang H, Li B, Ke M, Wang J, Dou J and Zhou C: The cathelicidin-BF Lys16 mutant Cbf-K16 selectively inhibits non-small cell lung cancer proliferation in vitro. Oncol Rep 30: 2502-2510, 2013.

22. Cheng YX, Liu R, Wang Q, Li BS, Xu XX, Hu M, Chen L, Fu Q, Pu DM and Hong L: Regular-induced apoptosis of cervical cancer cell line siha via cytochrome c release and caspase-3 and caspase-9 activation. Chin J Integr Med 18: 359-365, 2012.
23. Lu J, Yao YY, Dai QM, Ma GS, Zhang SF, Cao L, Ren LQ and Liu NF: Erythropoietin attenuates cardiac dysfunction by increasing myocardial angiogenesis and inhibiting interstitial fibrosis in diabetic rats. Cardiovasc Diabetol 11: 105, 2012.

24. Han YY, Liu HY, Han DJ, Zong XC, Zhang SQ and Chen YQ: Role of glycosylation in the anticancer activity of antibacterial peptides against breast cancer cells. Biochem Pharmacol 86: 1254-1262, 2013.

25. Tseng TH, Shen CH, Huang WS, Chen CN, Liang WH, Lin TH and Kuo HC: Activation of neutral-sphingomyelinase, MAPKs and p75 NTR-mediating caffeic acid phenethyl ester-induced apoptosis in C6 glioma cells. J Biomed Sci 21: 61, 2014.

26. Floyd DH, Zhang Y, Dey BK, Kefas B, Breit H, Marks K, Dutta A, Herold-Mende C, Synowitz M, Glass R, et al: Novel anti-apoptotic microRNAs $582-5$ p and 363 promote human glioblastoma stem cell survival via direct inhibition of caspase 3, caspase 9 and Bim. PLoS One 9: e96239, 2014.

27. Wyllie AH: 'Where, O death, is thy sting?' A brief review of apoptosis biology. Mol Neurobiol 42: 4-9, 2010.

28. Nagase H and Woessner JF Jr: Matrix metalloproteinases. J Biol Chem 274: 21491-21494, 1999.

29. Kim YS, Kim SH, Kang JG and Ko JH: Expression level and glycan dynamics determine the net effects of TIMP-1 on cancer progression. BMB Rep 45: 623-628, 2012.

30. Kim HR, Kim JM, Kim MS, Hwang JK, Park YJ, Yang SH, Kim HJ, Ryu DG, Lee DS, Oh H, et al: Saussurea lappa extract suppresses TPA-induced cell invasion via inhibition of NF- $\kappa \mathrm{B}-$ dependent MMP-9 expression in MCF-7 breast cancer cells. BMC Complement Altern Med 14: 170, 2014. 